\title{
Correlation of Depression, Anxiety and Stress with Quality of Life in COVID-19 Pandemic
}

\section{Aysha Rashid, ${ }^{1}$ Umair Mudassar, ${ }^{2}$ Ismail Tariq, ${ }^{3}$ Adil Zaheer, ${ }^{4}$ Mariam Iftikhar, ${ }^{5}$ Nauman Mazhar $^{6}$}

\begin{abstract}
Objectives: To determine correlation between depression, anxiety, stress, and quality of life among adults in Covid-19 and evaluate the impact of demographics on quality of life.

Methods: It was a cross-sectional study carried out at a tertiary care hospital. Patients presenting in the psychiatry outdoor of age 18 to 60 years, of both genders and scoring $\geq 21$ on Depression, Anxiety, and Stress Scale (DASS) were enrolled in the study and depression, anxiety and stress severity was assessed and Quality of Life Scale (QOLS) was applied on all to assess their quality of life. All findings were then subjected to statistical analysis.

Results: The mean age of the patients was $21.78 \pm 3.204$, mean depression score on DASS was $8.58 \pm 4.510$, mean anxiety score on DASS was $11.68 \pm 4.160$ and the mean stress score on DASS was $14.84 \pm 3.192$. There were $63.5 \%$ males and $36.5 \%$ females. Depression, anxiety and stress had a negative correlation with quality of life. Depression and stress were significantly correlated negatively with quality of life $(p=0.000)$. No demographical factor was significantly associated with poor quality of life.

Conclusion: Depression, anxiety and stress were negatively correlated with poorer QOL and depression and stress had significant association with poor QOL.

Key words: Anxiety, Depression, Covid-19, Quality of life

How to cite: Rashid A., Mudassar U., Tariq I., Zaheer A., Iftikhar M., Mazhar N. Correlation of Depression, Anxiety and Stress with Quality of Life in COVID-19 Pandemic. Esculapio 2021;17(02):195-199.

DOI: https://doi.org/10.51273/esc21.2517218
\end{abstract}

\section{Introduction}

$\mathrm{D}$ uring the pandemic of COVID-19, more than 100,000 deaths have been reported in United States till date ${ }^{1,2}$. Besides prevailing fears of having the virus, the level of stress has been raised because of other factors as well, such as confinement and social isolation in order to reduce the spread of virus. ${ }^{3,4}$ The adaptation to this whole new lifestyle made people

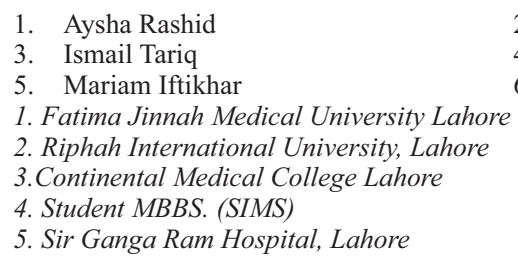

5. Sir Ganga Ram Hospital, Lahore

Correspondence:

Aysha Rashid, Associate Professor and HOD Fatima Jinnah Medical University Lahore. Email: ashi.32c@gmail.com uncertain and confused and it made it difficult for them to maintain their psychological well-being as many individuals had been grieving over losing their loved ones during this pandemic, losing their job and other opportunities professionally and had difficulty as well as delay in achieving the major goals in life. ${ }^{5,6,7}$ Evidence from previous literature suggests that worldwide among different populations, the prevalence of depression and anxiety rapidly rose in the initial time of the pandemic, ${ }^{8}$ with an estimated prevalence rate of $20 \%$ and $35 \%$ respectively. ${ }^{9}$ Furthermore, fearfulness, worry and stress as well as stigma and fear towards people who are being suspected to have Covid-19 infection have been reported to have negative effect on an individual's life. ${ }^{10,11}$ Additionally, because of limited social access and restricted activities, that usually help in maintaining psychological health, psychiatric distress may get exacerbated, thus affecting 
functioning and quality of life of people $\mathrm{e}^{12,13}$. In as study by Salman M, (2020) on university students of Lahore, it was found that during Covid-19 pandemic moderate to severe anxiety and depression was found in $34 \%$ and $45 \%$ participants respectively. Similar study revealed that the major cause of psychological distress among the participants was the negative affect of ongoing pandemic on their daily life $(p<0.001) .{ }^{14}$ In another study, Amin F (2020) revealed that $43 \%$ of the Pakistani frontline physicians suffered from anxiety/ depression during this pandemic. ${ }^{15}$ Ashraf F (2021) revealed that during Covid-19 pandemic in Pakistan, psychological distress was less common in people who had high level of satisfaction with life and had a meaning in life $(\mathrm{z}=-3.507, \mathrm{p}<0.0001$ and $\mathrm{z}=-2.632, \mathrm{p}<0.001$, respectively)16. In a study, it was revealed that patients who were diagnosed to have mental health problems during Covid-19 were more prone to have impaired quality of life i.e. in terms of physical and mental functioning and this association was found to be significant $(\mathrm{P}<0.001) .{ }^{17}$ In another study by Zhang Y, (2020) assessed the effect of Covid-19 on mental health and quality of life of patients and it was found that $52.1 \%$ participants had mental health related issue and 7.6\% had negative impact on the quality of life of the patients and the association between these two was significant $(\mathrm{p}<0.05){ }^{18}$

Majority of the international and local researches conducted so far focused on adverse outcome related to this pandemic and shifted their attention towards evaluating more positive and preventive outcomes of this pandemic, such as quality of life. However, there is still a need of conducting further local research for confirmation of the psychological factors as well as demographical factors that can affect quality of life of patients during this pandemic in order to confirm the reliability of the aforementioned results. In light of this, the current study aimed to determine the relation between depression, anxiety, stress and quality of life of individuals in Pakistan during this COVID-19 pandemic and determine the effect of demographical profile on quality of life of individuals. This study will provide guidance about emerging psychological issues and their effect on quality of life of individuals, which if addressed earlier and properly can help in reducing morbidity associated with the condition and can improve the overall quality of life of people.

\section{The aim of the study}

The study aimed to examine the correlation between depression, anxiety, stress, and quality of life among young adults during the COVID-19 pandemic and the association of demographical factors with quality of life.

\section{Methods}

It was a cross-sectional study. After taking approval from the ethical review committee, a total of 274 patients of both genders, aged 18 to 60 years who presented to the outdoor of Psychiatry Department in a tertiary care hospital were enrolled if they fulfilled the inclusion criteria i.e. on Depression, Anxiety and Stress scale their overall score was 21 and the symptoms specifically started during the Covid-19 pandemic in Pakistan i.e. from March, 2020 onwards. They were ensured that their data will be kept confidential and that data is only used for current research. Participants had the right without penalty to leave this study. Written informed consent was taken from all the patients. Patients with other mental health related issues such as schizophrenia, obsessive-compulsive disorder, drug abuse or organicity were excluded from the study.

Demographical detail, clinical history and details according to DASS scale were enquired from all patients and findings were noted down on a predesigned performa. All patients were then given Quality of Life Scale (QOLS) for assessing quality of life. ${ }^{2}$ In QOLS, the seven responses were $(7=$ delighted $),(6=$ pleased $),(5=$ mostly satisfied), (4=mixed), (3=mostly dissatisfied $)$, (2=unhappy), (1=terrible). The QOLS scores ranged from 16 to 112 . The QOLS scores were summed and higher score indicated a higher quality of life with a cut off of 80 representing good quality of life. ${ }^{2}$

DASS scale has 7 domains each divided into subscales to assess depression, anxiety and stress ${ }^{2}$. Using DASS, the severity of depression was categorized as no depression (0-9), mild (score 10-12), moderate (13-20), severe (21-27) and extremely severe ( $>28$ score). The severity of anxiety was categorized as no anxiety (06), mild (score 7-9), moderate (10-14), severe (15-19) and extremely severe ( $>20$ score). The severity of stress was categorized as no stress (0-10), mild stress (score 11-18), moderate stress (19-26), severe stress (27-34) and extremely severe stress ( $>34$ score). All findings were noted down on a predesigned performa and was subjected to statistical analysis. ${ }^{2}$

Data was analyzed using SPSS version 24.00. Quantitative variables such age, DASS score for depression, 
Table 1: Showing Mean of Quantitative Variables

\begin{tabular}{lc}
\hline \multicolumn{1}{c}{ Quantitative Variables } & $\begin{array}{c}\mathbf{N}=\mathbf{2 7 4} \\
\text { Mean }\end{array}$ \\
\hline $\mathrm{Ag}$ & $45.72 \pm 19.2$ \\
DASS Score: & \\
\multicolumn{1}{c}{ Depression } & $8.58 \pm 4.510$ \\
$\quad$ Anxiety & $11.68 \pm 4.160$ \\
Stress & $14.84 \pm 3.192$ \\
Quality of life Scale Score & $75.08 \pm 21.049$ \\
\hline
\end{tabular}

Table 2: Showing Frequency Distribution of Qualitative Variables

\begin{tabular}{|c|c|}
\hline Qualitative Variables & $\begin{array}{c}\mathrm{N}=274 \\
\text { Frequency (Percentage) }\end{array}$ \\
\hline \multicolumn{2}{|l|}{ Age groups: } \\
\hline Young age (18 to 30 years) & $53(19.3 \%)$ \\
\hline Early middle age ( 31 to 45 years) & $148(54 \%)$ \\
\hline Late middle age (46 to 60 years) & $73(26.6 \%)$ \\
\hline \multicolumn{2}{|l|}{ Gender: } \\
\hline Male & $174(63.5 \%)$ \\
\hline Female & $100(36.5 \%)$ \\
\hline \multicolumn{2}{|l|}{ Family system: } \\
\hline Nuclear & $163(59.5 \%)$ \\
\hline Joint & $111(40.5 \%)$ \\
\hline \multicolumn{2}{|l|}{ Place of living } \\
\hline Urban & $168(61.3 \%)$ \\
\hline Rural & $106(38.7 \%)$ \\
\hline \multicolumn{2}{|l|}{ Depression: } \\
\hline Mild depression & $50(18.3 \%)$ \\
\hline Moderate depression & $116(42.3 \%)$ \\
\hline Severe depression & $53(19.3 \%)$ \\
\hline Extremely severe depression & $55(20.1 \%)$ \\
\hline \multicolumn{2}{|l|}{ Anxiety: } \\
\hline Mild anxiety & $23(8.4 \%)$ \\
\hline Moderate anxiety & $92(33.6 \%)$ \\
\hline Severe anxiety & $70(25.5 \%)$ \\
\hline Extremely severe anxiety & $89(32.5 \%)$ \\
\hline \multicolumn{2}{|l|}{ Stress: } \\
\hline Mild stress & $22(8 \%)$ \\
\hline Moderate stress & $11(4 \%)$ \\
\hline Severe stress & $47(17.2 \%)$ \\
\hline Extremely severe stress & $194(70.8 \%)$ \\
\hline \multicolumn{2}{|l|}{ Quality of Life: } \\
\hline Poor & $144(52.6 \%)$ \\
\hline Good & $130(47.4 \%)$ \\
\hline
\end{tabular}

anxiety and stress and QOLS score was presented as mean and standard deviation. Qualitative data such as gender, family system, place of living, profession, severity of depression, severity anxiety, severity of stress and quality of life were presented as frequency and percentage. Data was stratified for age, gender, family system and place of living. Post-stratification chi square test was applied and a $p$ value of 0.05 was considered significant. The relationship between depression, anxiety, stress, and quality of life among young adults during the COVID-19 pandemic was determined by Pearson correlation test and a $\mathrm{p}$ value of 0.05 was considered significant.

\section{Results}

The mean age of the patients was $21.78 \pm 3.204$, mean depression score on DASS was $8.58 \pm 4.510$, mean anxiety score on DASS was $11.68 \pm 4.160$ and the mean stress score on DASS was 14.84 \pm 3.192 (Table 1). The mean QOLS score was 75.08 \pm 21.049 (Table 1). Frequency distribution of qualitative variables is presented in table 2. Association between quality of life and

Table 3: Showing Assocition Of Demographical Factors And Depression, Anxiety And Stress With Quality OfLife

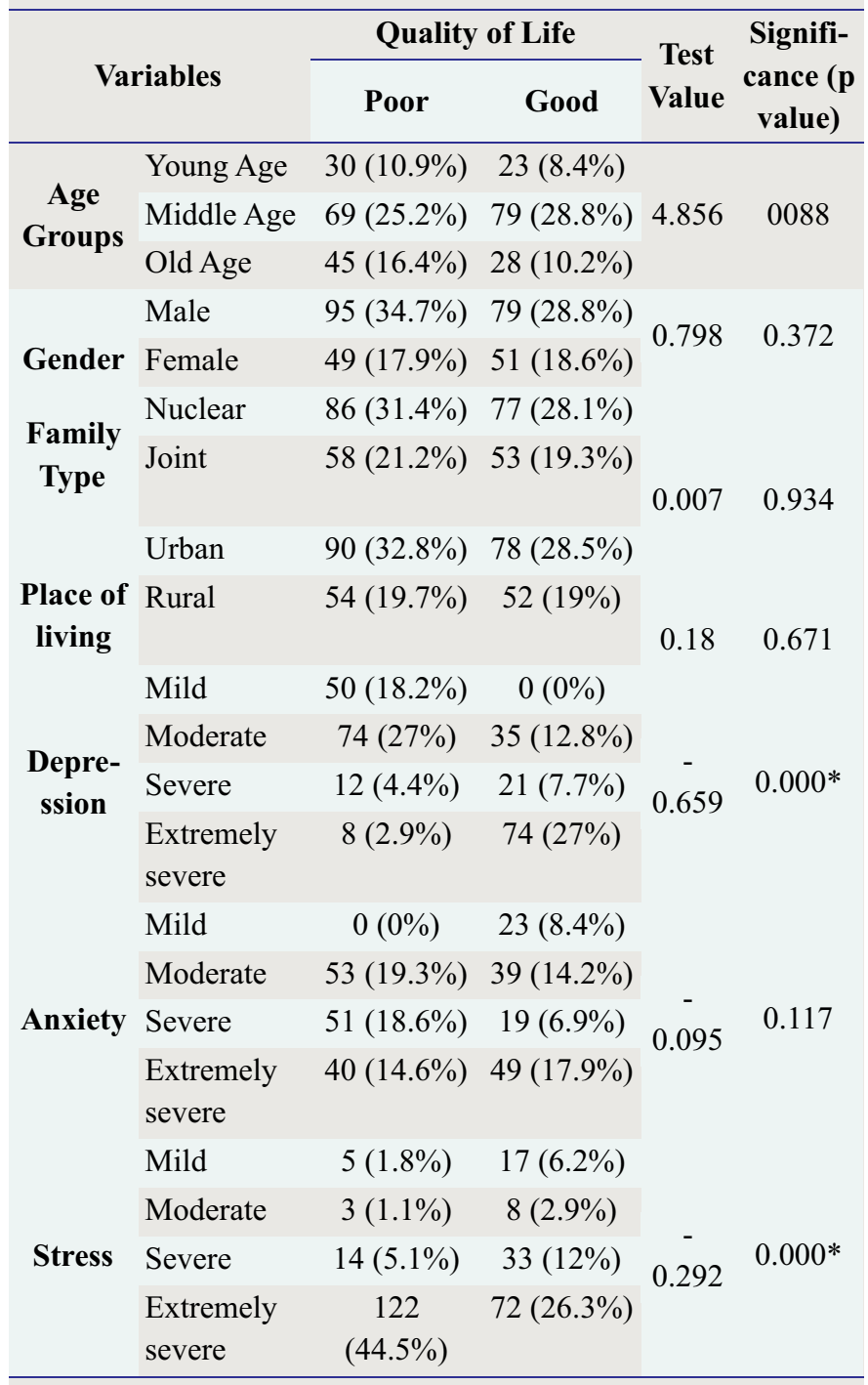

${ }^{*} \mathrm{p}$ value $\leq 0.05$ was considered significant 
demographical factors, depression, anxiety and stress are presented in table 3.

\section{Discussion}

The current study revealed that depression, anxiety and stress were negatively correlated with quality of life i.e. presence of these mental health issues were associated with a poor quality of life. However, only correlation of depression and stress with quality of life was found to be statistically significant. Demographical factors such as age, gender, family type and place of residence were not found to be significantly associated with poorer quality of life.

Globally, concerns have been expressed deeply by the researchers about a high prevalence of issues related to mental health in both general and vulnerable population. ${ }^{14,15}$ It is necessary to determine these problems in order to plan things accordingly. ${ }^{16,17}$ The current research was in accordance with the call of the researchers to provide an overview of prevailing mental health issues in COVID-19 pandemic in Pakistan and to see the impact on quality of life of people. It is difficult to make comparison of findings of this research with the previous ones carried out during non-pandemic era owing to the fact that the difficulties faced by individuals during COVID-19 are inconsistent in comparison to the non-pandemic times. The study revealed that depression and stress had significant correlation with poorer quality of life. Thus, higher scores of depression and stress were associated with low scores on quality of life scale thus denoting that these mental health issues lead to poor quality of life in participants during COVID-19 pandemic. In a study by Zhang et al. in 2020, it was found that mental issues related to Covid-19 had only mild impact on the quality of life of individuals and only $7.6 \%$ had poorer quality of life as assessed by Impact of event scale (IES), however, this association was found to be significant $(\mathrm{p}<$ $0.005) .{ }^{18}$ Our study revealed a higher percentage of poorer quality of life in individuals who had mental health issues i.e. 52.6\%. In another study Algahtani et al. showed that patients with depression $(\mathrm{OR}=5.70)$, anxiety $(\mathrm{OR}=5.47)$ and stress $(\mathrm{OR}=6.57)$ had scored on the lower side of the QOL scale and thus were associated with poorer quality of life. An important component of overall QOL is psychological dimension. Study by Algahtani et al. validated the relationship between quality of life and psychological health of patients ${ }^{2}$. Similar findings were reported by another study con- ducted in Saudia Arabia, which revealed that individuals who were highly distressed reported to have poor health related quality of life. ${ }^{23}$ These findings were in line with the findings of current study which too revealed that poor quality of life was associated with depression and stress, however, anxiety was not significantly linked up.

The situation of COVID-19 has not changed much and still uncertainty exists about its effects and outcomes. ${ }^{18,19}$ Looking at the results of current research, mental health issues specially related to depression and stress must be addressed properly, as these mental health issues can have serious consequences and thus necessitates that measures should be taken adequately and promptly to reduce mental health issues and improve quality of life of individuals during this pandemic.

The study had certain limitations. Firstly, it was carried out at a single center and the sample size was small, so the results cannot be generalized. Secondly, the effect of providing treatment to the patients presenting with depression, anxiety and stress was not assessed in terms of improvement in quality of life. Thirdly, besides pandemic and demographical details, other factors leading to depression, anxiety, stress and poorer quality of life were not assessed.

\section{Conclusion}

COVID-19 pandemic is associated with significant mental health issues such as depression and stress that lead to poor quality of life. Adequate attention should be given to these mental health issues to ensure proper functioning and quality of life of individuals by screening individuals earlier and provision of prompt psychological services to those in need.

\section{Conflict of Interest: None}

\section{References}

1. Shamblaw AL, Rumas RL, Best MW. Coping during the COVID-19 pandemic: Relations with mental health and quality of life. Canadian Psychology. 2021 Jan;62(1):92-100.

2. Algahtani FD, Hassan SU, Alsaif B, Zrieq R. Assessment of the quality of life during COVID-19 pandemic: A cross-sectional survey from the kingdom of Saudi Arabia. International Journal of Environmental Research and Public Health. 2021 Jan;18(3):847.

3. Zhang HH, Zhao YJ, Wang C, Zhang Q, Yu HY, Cheung $\mathrm{T}$, et al. Depression and its relationship with quality of life in frontline psychiatric clinicians during the COVID19 pandemic in China: a national survey. International 
Journal of Biological Sciences. 2021;17(3):683.

4. Daneshfar Z, Sadatmahalleh SJ, Youseflu S, Khomami MB, Kazemnejad A. Influential factors on quality of life in married Iranian women during the COVID-19 pandemic in 2020: a path analysis. BMC women's health. 2021 Dec;21(1):1-8.

5. Ravens-Sieberer U, Kaman A, Erhart M, Devine J, Schlack R, Otto C. Impact of the COVID-19 pandemic on quality of life and mental health in children and adolescents in Germany. European Child \& Adolescent Psychiatry. 2021 Jan;25:1-1.

6. Leibovitz T, Shamblaw AL, Rumas R, Best MW. COVID-19 conspiracy beliefs: Relations with anxiety, quality of life, and schemas. Personality and Individual Differences. 2021 Jun 1;175:110704.

7. Wang C, Pan R, Wan X, Tan Y, Xu L, McIntyre RS, et al. A longitudinal study on the mental health of general population during the COVID-19 epidemic in China. Brain, behavior, and immunity. $2020 \mathrm{Jul}$ 1;87:40-8.

8. Korkmaz S, Kazgan A, Çekiç S, Tartar AS, Balcı HN, Atmaca M. The anxiety levels, quality of sleep and life and problem-solving skills in healthcare workers employed in COVID-19 services. Journal of Clinical Neuroscience. 2020 Oct 1;80:131-6.

9. Lakhan R, Agrawal A, Sharma M. Prevalence of Depression, Anxiety, and Stress during COVID-19 Pandemic. Journal of Neurosciences in Rural Practice. 2020 Oct; 11(4):519.

10. Epifanio MS, Andrei F, Mancini G, Agostini F, Piombo MA, Spicuzza V, et al. The impact of COVID-19 pandemic and lockdown measures on quality of life among Italian general population. Journal of Clinical Medicine. 2021 Jan;10(2):289.

11. Abdullah MF, Yusof HA, ShariffNM, Hami R, Nisman NF, Law KS. Depression and anxiety in the Malaysian urban population and their association with demographic characteristics, quality of life, and the emergence of the COVID-19 pandemic. Current Psychology. 2021 Feb 19:1-2.

12. Levkovich I, Shinan-Altman S, Essar Schvartz N, Alperin M. Depression and Health-Related Quality of Life Among Elderly Patients during the COVID19 Pandemic in Israel: A Cross-sectional Study. Journal of Primary Care \& Community Health. 2021 Feb; 12: 2150132721995448.

13. Lipskaya-Velikovsky L. COVID-19 Isolation in Healthy Population in Israel: Challenges in Daily Life, Mental Health, Resilience, and Quality of Life. International Journal of Environmental Research and Public Health. 2021 Jan;18(3):999.

14. Salman, M., Asif, N., Mustafa, Z.U., Khan, T.M., Shehzadi, N. and Hussain, K., Psychological Impact of COVID-19 on Pakistani University Students and How They are Coping. Medrxiv. 2020: 1-16.
15. Amin F, Sharif S, Saeed R, Durrani N, Jilani D. COVID19 pandemic-knowledge, perception, anxiety and depression among frontline doctors of Pakistan. BMC psychiatry. $2020 \mathrm{Dec} ; 20(1): 1-9$.

16. Ashraf F, Zareen G, Nusrat A, Arif A, Griffiths MD. Correlates of Psychological Distress Among Pakistani Adults During the COVID-19 Outbreak: Parallel and Serial Mediation Analyses. Frontiers in Psychology. 2021 Mar 31;12:825.

17. Liu CH, Stevens C, Conrad RC, Hahm HC. Evidence for elevated psychiatric distress, poor sleep, and quality of life concerns during the COVID-19 pandemic among US young adults with suspected and reported psychiatric diagnoses. Psychiatry research. 2020 Oct 1;292: 113345.

18. Zhang Y, Ma ZF. Impact of the COVID-19 pandemic on mental health and quality of life among local residents in Liaoning Province, China: A cross-sectional study. International journal of environmental research and public health. $2020 \mathrm{Jan} ; 17(7): 2381$.

19. Ma YF, Li W, Deng HB, Wang L, Wang Y, Wang PH, et al. Prevalence of depression and its association with quality of life in clinically stable patients with COVID19. Journal of affective disorders. 2020 Oct 1;275: 145-8.

20. Zahra D, Sadatmahalleh SJ, Samaneh Y, Mahnaz BK, Anoshiravan K. Influential Factors on quality of life in married Iranian women during the Covid-19 pandemic in 2020: A Path Analysis.

21. Cam HH, Top FU, Ayyildiz TK. Impact of the COVID19 pandemic on mental health and health-related quality of life among university students in Turkey. Current Psychology. 2021 Mar 31:1-0.

22. Korkmaz S, Kazgan A, Çekiç S, Tartar AS, Balcı HN, Atmaca M. The anxiety levels, quality of sleep and life and problem-solving skills in healthcare workers employed in COVID-19 services. Journal of Clinical Neuroscience. 2020 Oct 1;80:131-6.

23. Hawash MM, Alhazmi AH, Wafik W, Muzammil K, Mushfiq S, Ahmed HA. The Association of COVID19 Pandemic Stress With Health-Related Quality of Life in the Kingdom of Saudi Arabia: A Cross-Sectional Analytical Study. Frontiers in Public Health. 2021;9.

\section{Authors Contribution}

A.R: Conceptualization of Project

U.M: Statistical Analysis

I.T: Writing of Manuscript

A.Z: Data Collection

M.I: Literature Search

N.M: Drafting, RevisionAuthors 